\title{
Avaliação da estabilidade do biodiesel produzido a partir da Moringa oleifera lam
}

Biodiesel stability assessment produced from Moringa oleifera lam during storage

\author{
L. C. T. Andrade ${ }^{1 *}$; F. R. M. França2; A. L. D. Ramos³; G. F. Silva ${ }^{4}$ \\ ${ }^{1}$ Departamento de Engenharia Química/Laboratório de Tecnologias Alternativas/Universidade Federal de Sergipe, \\ 49100-000, São Cristóvão-Sergipe, Brasil \\ ${ }^{2}$ Departamento de Engenharia Química/Laboratório de Catálise/Universidade Federal da Bahia, 40210-730, \\ Salvador-Bahia, Brasil
}

${ }^{3}$ Núcleo de Engenharia Ambiental/Laboratório de Tecnologias Alternativas/Universidade Federal de Sergipe, 49100000, São Cristóvão-Sergipe, Brasil

${ }^{4}$ Núcleo de Graduação em Engenharia de Petróleo/Laboratório de Tecnologias Alternativas/Universidade Federal de Sergipe, 49100-000, São Cristóvão-Sergipe, Brasil

*liviacaroline1987@yahoo.com.br

(Recebido em 14 de março de 2016; aceito em 11 de abril de 2016)

\begin{abstract}
Os processos oxidativos ocorrem devido às temperaturas elevadas e à presença de ácidos graxos insaturados, com isso, torna-se um desafio a produção de biodiesel de qualidade. Neste sentido, o presente trabalho apresenta parâmetros indicativos da influência antioxidante do biodiesel obtido a partir da Moringa oleifera Lam em diferentes percentuais em biodiesel de soja. As amostras foram caracterizadas através de análises físico-químicas e mediante o estudo oxidativo, através do método Rancimat. As amostras de biodiesel de soja foram aditivadas com o antioxidante nas concentrações de 100, 500, 1000, 2000, 3000, 4000 e $5000 \mathrm{ppm}$. Todas as amostras foram analisadas no tempo zero. Os resultados obtidos, mostraram que o tempo de estabilidade oxidativa ultrapassou o limite estabelecido pela ANP (mínimo de 8horas) somente a partir da concentração de 2000 ppm e revelaram que quanto maior a concentração de antioxidante utilizada, maior é a estabilidade oxidativa.
\end{abstract}

Palavras-Chave: Biodiesel; Estabilidade Oxidativa; Antioxidante.

The oxidative processes occur due to high temperatures and the presence of unsaturated fatty acids, making a challenge to keep the quality of the biodiesel produced. In this sense, this paper presents data about the antioxidant influence of biodiesel obtained from the Moringa oleifera Lam mixed in different percentages with soybean biodiesel, the most used oil for biodiesel production in Brazil $(81 \%)$. The samples were characterized about oxidative stability by the Rancimat method, Concentrations of 100, 500, 1000, 2000, 3000,4000 and $5000 \mathrm{ppm}$. All samples were analyzed at time zero. The results obtained showed that the oxidative stability time exceeded the limit established by ANP (minimum $8 \mathrm{~h}$ ) only from the concentration of $2000 \mathrm{ppm}$, and revealed that the higher antioxidant concentration used, the greater the oxidative stability.

Keywords: Biodiesel; Oxidative Stability; Antioxidante.

\section{INTRODUÇÃO}

O biodiesel é um combustível já inserido na matriz energética brasileira, com incorporação de $7 \%$ ao diesel do petróleo. Uma atenção recente tem sido focada para os efeitos da oxidação causados pelo contato do biodiesel com o ar ambiente (autoxidação), reduzindo sua qualidade durante o armazenamento. Assim sendo, torna-se um desafio a produção de biodiesel de qualidade a partir de óleos e de suas misturas com combustíveis destilados do petróleo durante o longo período de estocagem.

O biodiesel produzido a partir do óleo de soja possui uma baixa estabilidade à oxidação, assim como o do óleo de girassol. A determinação da estabilidade oxidativa em laboratório baseia-se na metodologia de ensaio acelerado originalmente proposta por Hadorn e Zurcher [1]. A Agência 
Nacional de Petróleo, Gás Natural e Biocombustíveis (ANP), em sua Resolução nº 45 de 2014, estabelece um limite mínimo de estabilidade à oxidação de 8 horas ANP [2].

Segundo Sousa et al. [3], atualmente, estudos estão sendo realizados para aplicação de aditivos antioxidantes oriundos de fontes renováveis, adequados ao cenário ambiental atual e que tenham baixo valor agregado, uma vez que no Brasil gasta milhões de dólares por ano em aditivos sintéticos.

Por outro lado, a Moringa oleifera Lam é uma planta originária do nordeste indiano que, por ser amplamente utilizada em diversas áreas como na medicina, na agricultura, na alimentação e nas indústrias produtoras de cosméticos, tornou-se natural o seu plantio em diversos países. Além disso, a moringa possui um óleo de ótima qualidade, que, além de servir para a produção de biodiesel, possui uma excelente estabilidade à oxidação, ultrapassando o limite mínimo estabelecido pela Agência Nacional de Petróleo, Gás Natural e Biocombustíveis (ANP), chegando a atingir 93 horas de estabilidade ANP [2].

Esta pesquisa teve como objetivo avaliar a atividade antioxidante do aditivo proveniente da Moringa oleifera Lam, durante o armazenamento, em amostras de biodiesel com baixa estabilidade.

\section{MATERIAL E MÉTODOS}

\subsection{Obtenção e preparo do material oriundo da Moringa oleifera Lam}

Para fins de experimento a moringa utilizada foi de cultivo próprio da Universidade Federal de Sergipe, na Cidade Universitária "Professor José Aloísio de Campos", situada em São Cristóvão. Da moringa foram separadas as sementes com a casca, logo após cominuídas em um triturador e colocadas em estufa com circulação de ar sob temperatura de $40^{\circ} \mathrm{C}$, realizando-se um processo de secagem durante um período de 24 horas, tendo em vista que em testes preliminares a umidade entra em equilíbrio a partir de 20 horas.

\subsection{Desenvolvimento do antioxidante à base da Moringa oleifera Lam}

Conforme a metodologia utilizada por Pereira [4], o processo para obtenção do biodiesel da moringa (antioxidante), foi realizado pela rota de transesterificação in situ, utilizando-se álcool etílico, com proporção sementes/álcool [1:161 (m/v)], em mistura com um catalisador básico $(\mathrm{NaOH})$, juntamente com o pó das sementes da moringa com casca. Para esclarecimento destas etapas um diagrama de blocos é apresentado na Figura 1.

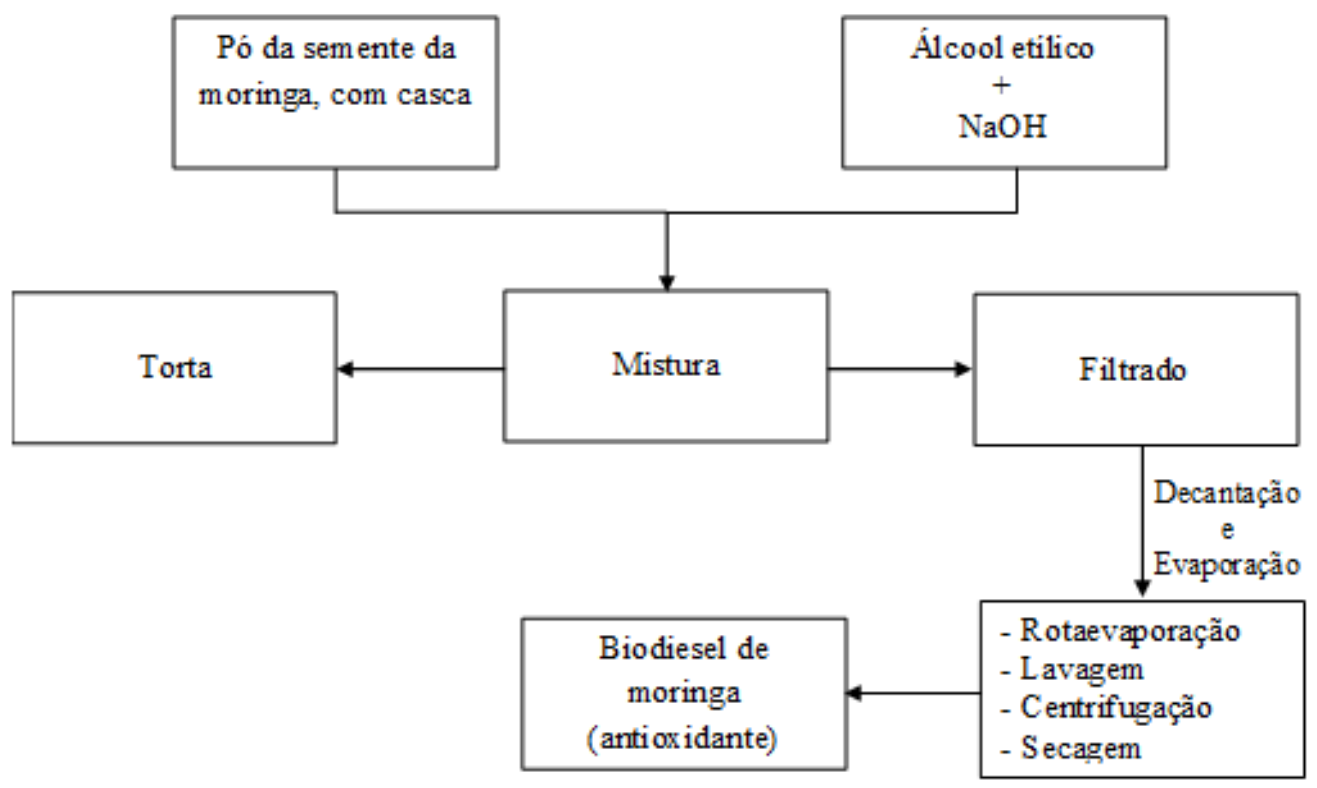

Figura 1: Diagrama do processo produtivo do biodiesel de moringa 
A partir do diagrama apresentado, torna-se relevante a abordagem das etapas para obtenção do composto. Na reação de transesterificação, foram adicionados $200 \mathrm{~g}$ do pó das sementes com casca e $6 \%$ em massa do catalisador básico. É importante salientar que a moringa contém um teor de óleo de 35 a $40 \%$ na semente com casca, ou seja, cerca de 70 a 80 g de óleo em cada extração.

O processo de mistura da solução de etanol com o catalisador foi conduzido em batelada por cerca de trinta minutos e, logo após, o pó das sementes foi adicionado ao misturador que realizou a homogeneização por $2,5 \mathrm{~h}$. Após esta etapa, a mistura foi separada por filtração a vácuo, resultando em um filtrado de biodiesel de moringa (antioxidante) e a torta. Em seguida, o filtrado permaneceu por 24 horas em um funil de separação para a retirada do glicerol.

Ainda com álcool, o filtrado foi colocado em rotaevaporador com temperatura igual a $45^{\circ} \mathrm{C}$, para que este fosse totalmente recuperado. O processo de lavagem ocorreu livre de álcool, com água destilada, à temperatura de $90^{\circ} \mathrm{C}$ e, logo após esta etapa, passou por um processo de centrifugação para eliminar sais e impurezas. Então, foi realizada a secagem para eliminar a umidade residual, em estufa a $100^{\circ} \mathrm{C}$, até atingir o limite estabelecido pela ANP de $200 \mathrm{mg} / \mathrm{kg}$, analisado através do método EN ISSO 12937.

\subsection{Técnicas de Caracterização do Biodiesel da Moringa}

Dentre os parâmetros físico-químicos foram realizadas análises conforme os métodos de determinação especificados na Tabela 1.

Tabela 1: Normas técnicas para caracterização do biodiesel de moringa (antioxidante)

\begin{tabular}{cc}
\hline Características & Resolução ANP \\
\hline Densidade a $20^{\circ} \mathrm{C}$ & ASTM D4052 \\
Viscosidade Cinemática a $40^{\circ} \mathrm{C}$ & EN ISO 3104 \\
Índice de Acidez, máx & EN 14104 \\
Ponto de Fulgor, máx & EN 3679 \\
Ésteres & EN 14103 \\
\hline
\end{tabular}

\subsection{Testes de Eficiência do Composto Aditivo da Moringa}

Para verificar a eficácia do antioxidante, foi realizado um estudo da estabilidade oxidativa com o uso do equipamento Rancimat, mostrado na Figura 2, instalado no LTA/UFS. Os óleos e gorduras são prematuramente envelhecidos pela decomposição oxidativa e os produtos formados por esta decomposição são separados por um fluxo de ar dentro de uma célula de medição abastecida por água deionizada. Uma suspensão foi preparada com cerca de $3 \mathrm{~g}$ de cada amostra e $50 \mathrm{~mL}$ de água deionizada, aquecidos a $110^{\circ} \mathrm{C}$. A condutividade da água deionizada, realizada pelo equipamento Rancimat, deve ser menor ou igual a $5 \mu \mathrm{S}$.

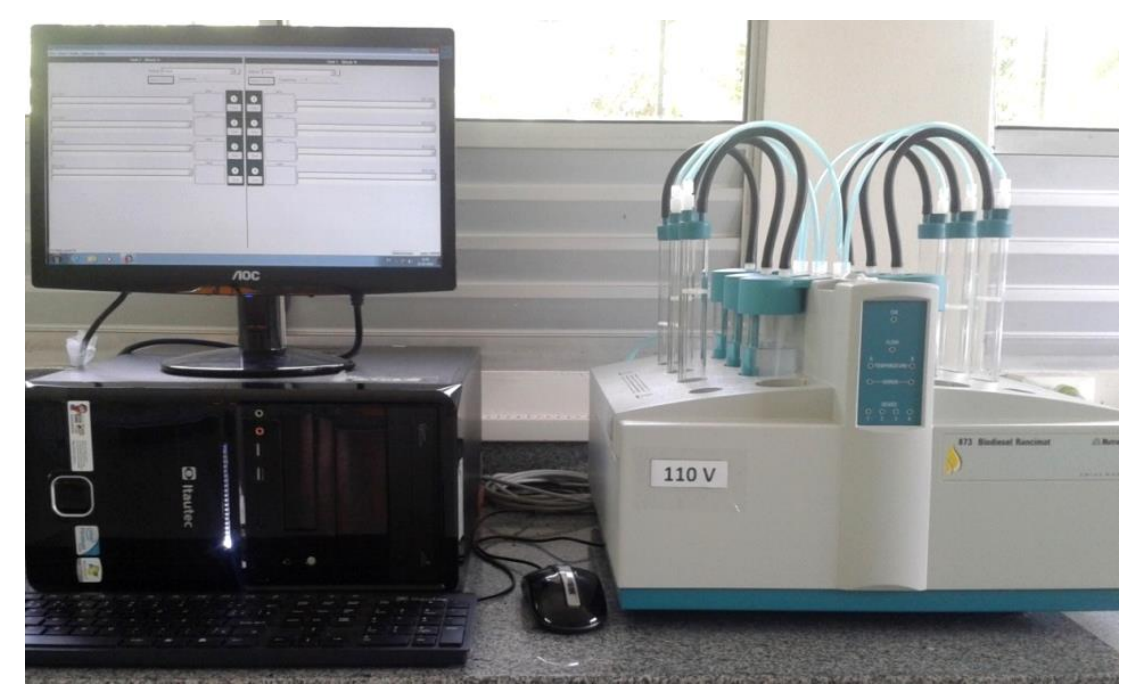

Figura 2: Equipamento Rancimat utilizado para medir a estabilidade à oxidação 


\section{RESULTADOS E DISCUSSÃO}

\subsection{Análises Físico-Químicas}

Na Tabela 2 constam os resultados da caracterização do biodiesel da moringa, utilizado como antioxidante. Além disso, constam também os resultados das análises realizadas para o biodiesel de soja e para a mistura soja+antioxidante (misturas).

Tabela 2: Caracterização físico-química

\begin{tabular}{ccccc}
\hline Características & $\begin{array}{c}\text { Biodiesel de } \\
\text { moringa } \\
\text { (antioxidante) }\end{array}$ & $\begin{array}{c}\text { Biodiesel de } \\
\text { soja }\end{array}$ & $\begin{array}{c}\text { Mistura } \\
(\mathbf{5 0 0 0} \mathbf{~ p p m})\end{array}$ & $\begin{array}{c}\text { Resolução } \\
\text { ANP }^{*}\end{array}$ \\
\hline $\begin{array}{c}\text { Densidade } \\
\left(\mathrm{kg} / \mathrm{m}^{3}\right) \text { a } 20^{\circ} \mathrm{C}\end{array}$ & 870 & 853 & 855 & $850-900$ \\
$\begin{array}{c}\text { Viscosidade } \\
\text { Cinemática } \\
\left(\mathrm{mm}^{2} / \mathrm{s}\right) \text { a } 40^{\circ} \mathrm{C}\end{array}$ & 6,43 & 4,30 & 5,03 & $3,0-6,0$ \\
$\quad \begin{array}{l}\text { Indice de } \\
\text { acidez }\end{array}$ & 0,059 & 0,051 & 0,057 & 0,50 (máx) \\
$(\mathrm{mL} / \mathrm{NaOH} / \mathrm{g})$ & 162 & 184 & 182 & 100 (mín.) \\
$\begin{array}{c}\text { Ponto de fulgor, } \\
{ }^{\circ} \mathrm{C}\end{array}$ & 58 & 98 & 90 & 96,5 (mín.) \\
\hline Ésteres $(\%)$ & & & &
\end{tabular}

Legenda: máx = valor máximo; $\min =$ valor mínimo

*Resolução n 45 de 2014

Com os resultados obtidos, fica evidente que o antioxidante apresentou densidade dentro das especificações estabelecidas, podendo ser utilizado também como combustível, sabendo-se que a alta densidade pode dificultar a injeção do combustível, reduzindo o rendimento do motor [5]. O valor da viscosidade do antioxidante apresentou-se, um pouco acima do intervalo permitido. De acordo com Oliveira et al. [6], este valor é influenciado pelas propriedades do ácido oléico presente em maior quantidade no óleo da moringa, que são promovidas pelas interações intermoleculares, assim como as forças de van der Walls, que se acentuam devido à molécula possuir grande massa molar, contribuindo para o aumento da viscosidade. Porém, quando utilizado como antioxidante no biodiesel de soja, este ocasionou uma pequena alteração, mas permanecendo dentro do intervalo que a norma estabelece. Este aumento é consistente com a mudança na composição química das misturas.

Os valores obtidos para o índice de acidez estão de acordo com a Norma, o que promove a minimização de reações indesejadas, como a saponificação ocasionada pelo meio básico do catalisador durante a reação de transesterificação. Os valores de ponto de fulgor também estão conforme a especificação permitida. Esta propriedade se torna importante quanto à segurança no manuseio e armazenamento do biodiesel, indicando a temperatura na qual surge a primeira observação de chama, uma vez que combustíveis com baixo ponto de fulgor indicam riscos com explosão. Para o biodiesel, os valores de ponto de fulgor são consideravelmente mais elevados que os valores encontrados para o diesel mineral. Como as temperaturas se apresentaram acima de $130^{\circ} \mathrm{C}$, fica dispensada a análise de álcool nas amostras.

A cromatografia gasosa determinou o percentual de ésteres do biodiesel de moringa (antioxidante), de soja e de suas misturas. A partir do resultado obtido para o antioxidante, foi possível observar que houve a conversão de apenas 58\% em ésteres, não sendo este valor representativo conforme estabelecido pela norma, restando em sua composição mono, di ou triglicerídeos. Esta baixa conversão, provavelmente, está atribuída à necessidade de otimização do processo produtivo, como a melhoria de alguns parâmetros, tais como temperatura, tempo e catalisador. Como o valor da blenda ficou abaixo da especificação, esta otimização e consequente aumento da conversão precisa ocorrer antes da mistura com o biodiesel de soja. 
Vale ressaltar que as quantidades de antioxidantes utilizadas não alteraram significativamente as características físico-químicas do biodiesel de soja, uma vez que o máximo de aditivo utilizado foi $5000 \mathrm{ppm}(\mathrm{v} / \mathrm{v})$ e que a presença destes compostos, devido à sua ação de minimizar a formação de produtos causadores da oxidação, conferem maior proteção às características analisadas.

\subsection{Avaliação da Influência Antioxidante do Biodiesel de Moringa Oleifera Lam}

A Figura 3 apresenta o perfil da estabilidade à oxidação do biodiesel de soja. Pode-se observar a baixa estabilidade deste biodiesel $(5,35 \pm 0,2 \mathrm{~h})$, abaixo do limite estabelecido pela ANP (mínimo de $8 \mathrm{~h}$ ), indicando a viabilidade de se estudar o uso de um composto aditivo antioxidante para aumentar esta estabilidade. Os valores de tempo de indução oxidativa para o óleo de soja, sem aditivos, está de acordo com o resultado encontrado por Cordeiro et al. [7], que relataram 5,2 horas nas mesmas condições de estudo. Esta baixa estabilidade oxidativa do óleo de soja é atribuída à elevada percentagem de ácidos graxos insaturados.

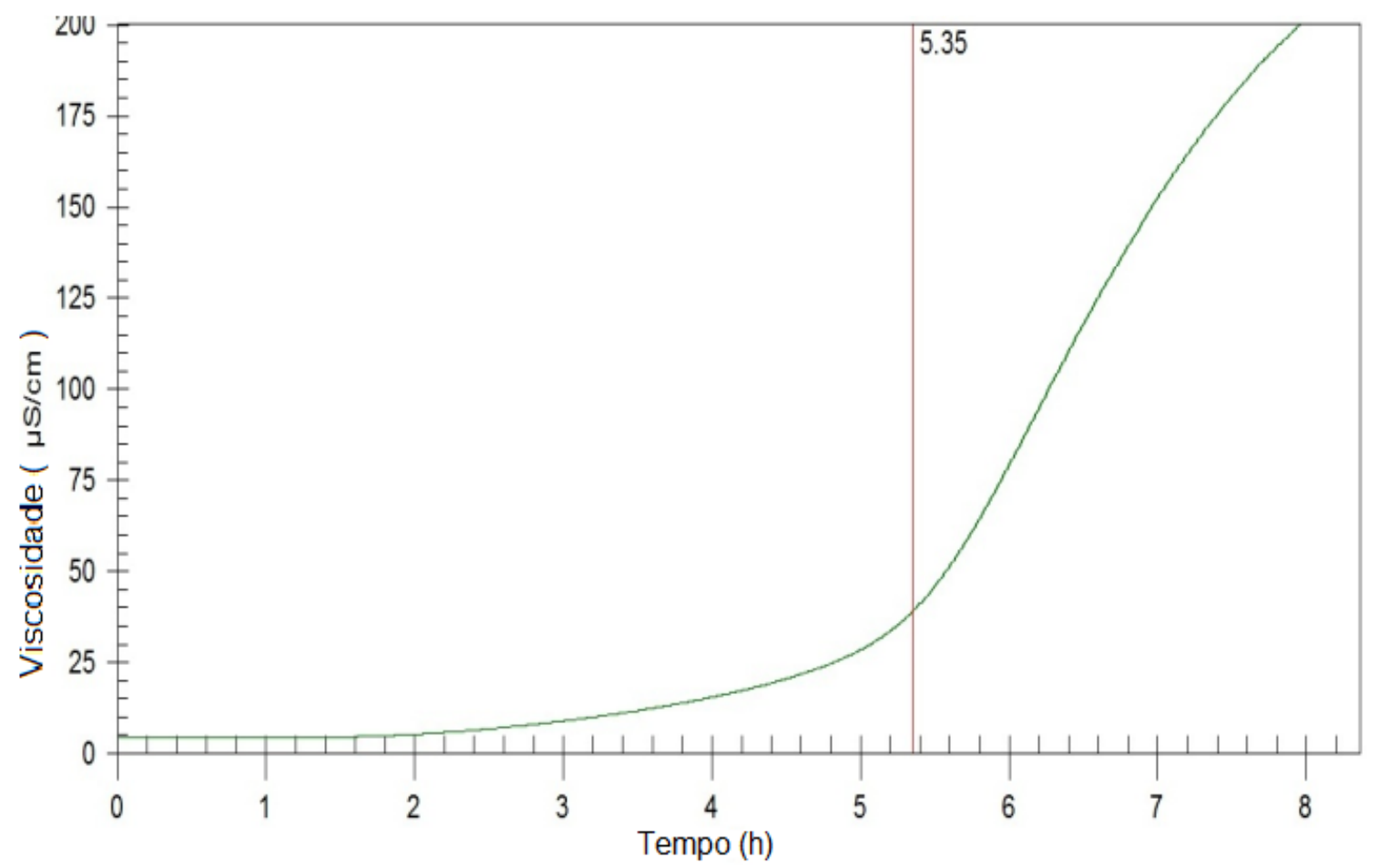

Figura 3: Tempo de estabilidade oxidativa do biodiesel de soja em função da viscosidade

A Figura 4 apresenta a influência da razão mássica do antioxidante obtido a partir da Moringa oleifera na estabilidade oxidativa do biodiesel de soja, constatando-se que, quanto maior a concentração de aditivo utilizada, maior o tempo de estabilidade à oxidação do biodiesel ao qual este for adicionado, conforme comprovado em estudo realizado por Taghvaei et al. [8]. 


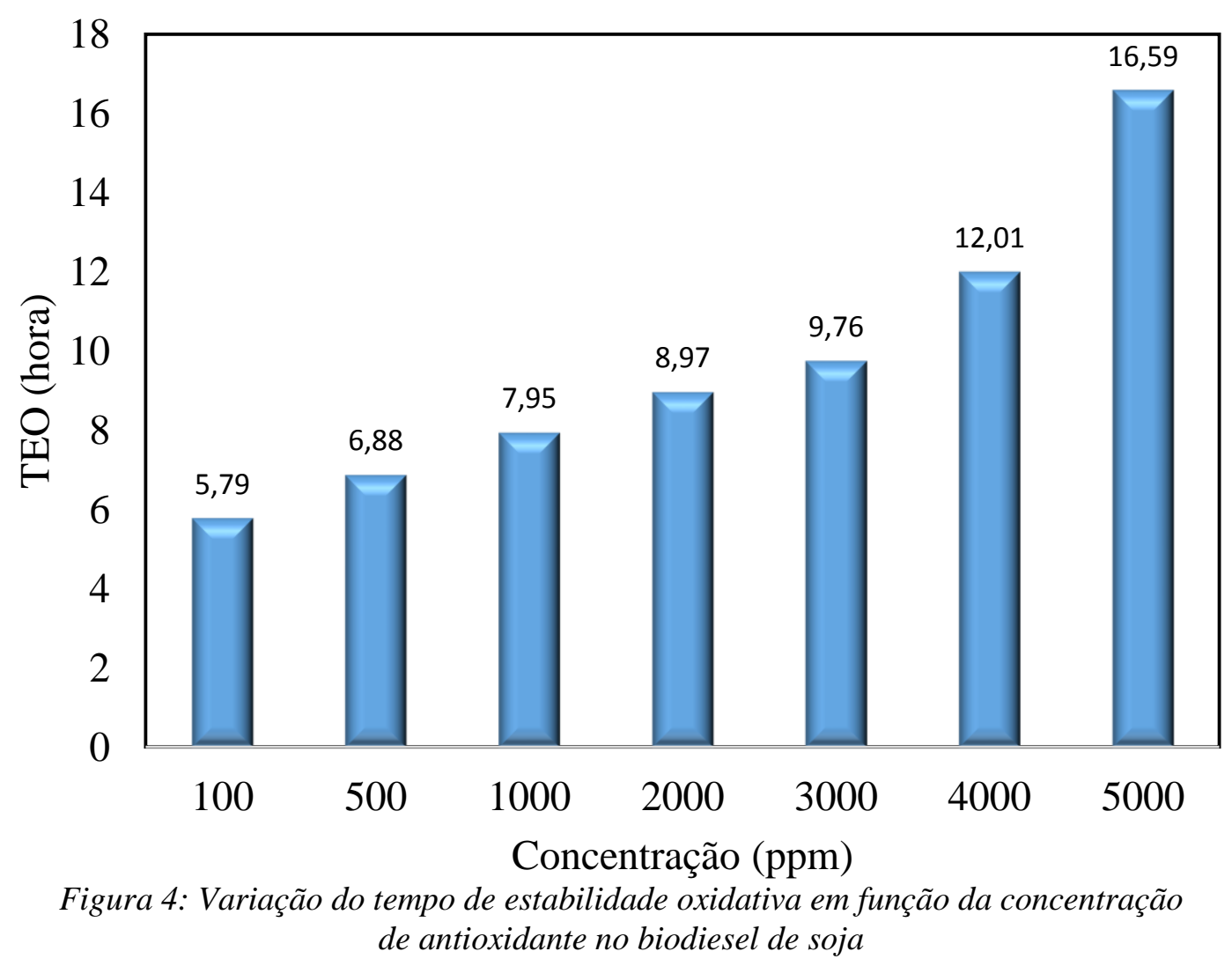

Mediante a adição da menor concentração de 100 ppm (v/v), a estabilidade saltou para 5,79 \pm 0,05 horas. Já na maior concentração de $5000 \mathrm{ppm}(\mathrm{v} / \mathrm{v})$, a estabilidade saltou para 16,59 \pm 0,02 horas, superando em mais de 8 horas o limite estabelecido pela ANP.

\section{CONCLUSÃO}

Com a finalização deste trabalho, observou-se a eficiência do biodiesel de Moringa oleifera Lam como antioxidante natural adicionado ao biodiesel de soja, no sentido de aumentar a estabilidade oxidativa de biodiesel com baixa estabilidade. Nas análises do tempo de estabilidade oxidativa via Rancimat, o tempo de indução do biodiesel de soja, inicialmente em 5,35 h, foi aumentado em $8 \%$ com a adição de 100 ppm do biodiesel de moringa (antioxidante). Somente, a partir da adição de 2000 ppm, as amostras apresentaram estabilidade acima de 8 horas, tempo mínimo estabelecido pela ANP.

\section{AGRADECIMENTOS}

LTA-Laboratório de Tecnologias Alternativas, PRH-45, FAPITEC/SE, FINEP e SERGIPETEC.

\section{REFERÊNCIAS BIBLIOGRÁFICAS}

1. Hadorn H, Zurcher K. Zurbestimmung der Oxydations stabilitat. Von Olenund Fetten. Deustsche Ledensmittel Rundschau, v. 70, n. 2, p. 57-65, 1974.

2. ANP - AGENCIA NACIONAL DE PETRÓLEO, GÁS NATURAL E BIOCOMBUSTÍVEIS. Resolução ANP no 45, de 28.11.2014 - DOU 26.08.2014.

3. Sousa LS, Moura CVR, Oliveira JE, Moura EM. Use of natural antioxidants in soybean biodiesel. Fuel (Guildford). 2014 Jun 14; 134: 420-428, doi: 10.1016/j.fuel.2014.06.007. 
4. Pereira DF. Potencialidades da Moringa oleifera Lam na Produção de Biodiesel e no Tratamento de Água Produzida na Extração de Petróleo. [dissertação]. Aracaju (SE): Universidade Federal de Sergipe; 2011. 87-97 p.

5. Santos AGD. Avaliação da estabilidade térmica do biodiesel de algodão, girassol, dendê e sebo bovino. [dissertação]. Natal-RN: Universidade Federal do Rio Grande do Norte; 2010. 57 p.

6. Oliveira DS, Fonseca XDS, Farias PN, Bezerra VS, Pinto CHC, Souza LD, Santos AGD, Matias LGO. Obtenção do biodiesel através da transesterificação do óleo de Moringa oleifera Lam. Holos. 2012 Mar 28; 1: 49-61, doi: http://dx.doi.org/10.15628/holos.2012.803.

7. Cordeiro AMTM, Medeiros ML, Silva MAAD, Silva IAA, Soledade LEB, Souza AL, Queiroz N, Souza AG. Rancimat and PDSC Accelerated Techniques for Evaluation of Oxidative Stability of Soybean Oil With Plant Extracts. J Therm Anal Calorim. 2013 Mar 3; 114: 827-832, doi: 10.1007/s10973-013-3036-0.

8. Taghvaei M, Jafari SM, Mahoonak AS, Nikoo AM, Rahmanian N, Javad H, Meshginfar N. The effect of natural antioxidants extracted from plant and animal resources on the oxidative stability of soybean oil. Food Sci Technol. 2014 Nov 5; 56: 124-130, doi: 10.1016/j.1wt.2013.11.009. 